\title{
Testicular Pain and Mesenteric Adenitis as an Atypical Presentation of COVID-19
}

\author{
Shreya Desai ${ }^{1}$, Dennis Citrin ${ }^{2}$, Mark Conneely ${ }^{3}$ \\ 1. Internal Medicine, Rosalind Franklin University of Medicine and Science, North Chicago, USA 2. Hematology and \\ Oncology, Captain James A. Lovell Federal Health Care Center, North Chicago, USA 3. Radiology and Nuclear Medicine, \\ Captain James A. Lovell Federal Health Care Center, North Chicago, USA
}

Corresponding author: Shreya Desai, shreya.desai@rosalindfranklin.edu

\begin{abstract}
A 21-year-old Caucasian male with no past medical history presented to the emergency department with right lower quadrant pain radiating to the right testicle for two days. He reported an occasional dry cough that day but denied any fever or other infectious symptoms. The patient was afebrile with a normal physical examination. CT of the abdomen and pelvis showed prominent right lower quadrant lymphadenopathy. Viral panel for common respiratory pathogens returned negative. A nasopharyngeal swab for SARS-CoV-2 by Xpert ${ }^{\circledR}$ Xpress SARS-CoV-2 reverse transcriptase-polymerase chain reaction (Cepheid Inc., Sunnyvale, CA) was positive. The patient remained in quarantine for 14 days. He was reevaluated seven weeks later with spontaneous resolution of his abdominal pain and the continued absence of upper respiratory symptoms. A repeat CT scan seven weeks later showed persistent mesenteric lymphadenopathy. Repeat COVID-19 testing was not performed at this time. While the frequency of atypical presentation of COVID-19 remains unknown, healthcare providers must continue to remain vigilant and consider COVID-19 as a differential diagnosis in any patient presenting to the emergency department despite the lack of respiratory and gastrointestinal symptoms. Further research is warranted to examine the possibility of asymptomatic spread in asymptomatic patients with persistent radiologic findings and to assess whether repeat COVID-19 testing is warranted in such patients.
\end{abstract}

Review began 05/17/2021 Review ended 06/02/2021 Published 06/27/2021

(c) Copyright 2021 Desai et al. This is an open access article distributed under the terms of the Creative Commons Attribution License CC-BY 4.0., which permits unrestricted use, distribution, and reproduction in any medium, provided the original author and source are credited.
Categories: Radiology, Gastroenterology, Infectious Disease

Keywords: covid-19, mesenteric adenitis, testicular pain, sars-cov-2 (severe acute respiratory syndrome coronavirus 2), asymptomatic covid-19

\section{Introduction}

The COVID-19 pandemic has caused a global health crisis that continues to ravage nations across the world. In the time of scarce medical supplies and personal protection equipment (PPE) shortage, asymptomatic or atypical presentations of COVID-19 can lead to unintentional exposure by the healthcare workers (HCW). There continues to be growing evidence regarding atypical presentations of COVID-19 infection. Here we present a case report of a man who was referred to the emergency department for testicular pain and tested positive for COVID-19 infection. He was subsequently found to have mesenteric lymphadenopathy which persisted for weeks after the resolution of the infection.

\section{Case Presentation}

A 21-year-old Caucasian male with no past medical history presented to the emergency department with right lower quadrant pain radiating to the right testicle for the past two days. He reported occasional dry cough that day but denied any fever, sore throat, rhinorrhea, body aches, vomiting, diarrhea, loss of taste or smell. He had no history of testicular torsion or abdominal trauma. The patient denied recent sick contacts. He was afebrile with normal vital signs. Examination of the lymph nodes, abdomen, and testes/scrotum was normal. Laboratory findings were unremarkable and did not show elevated inflammatory markers or lymphopenia. The computed tomography (CT) of the abdomen and pelvis showed prominent right lower quadrant lymphadenopathy (Figure 1,2). 


\section{Cureus}

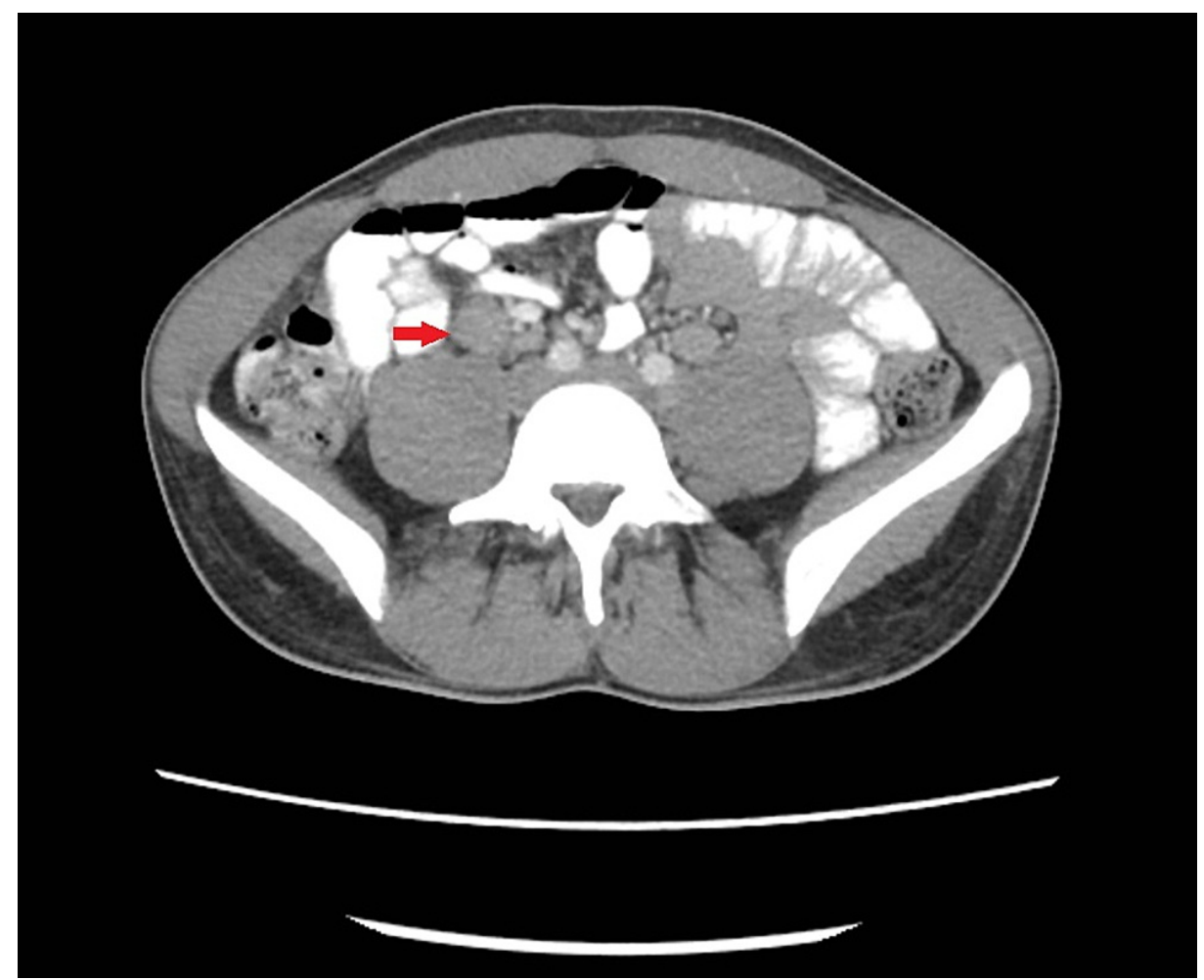

FIGURE 1: Axial image from CT of the abdomen and pelvis with oral and IV contrast demonstrates a cluster of enlarged mesenteric lymph nodes in the right lower quadrant (red arrow). 


\section{Cureus}

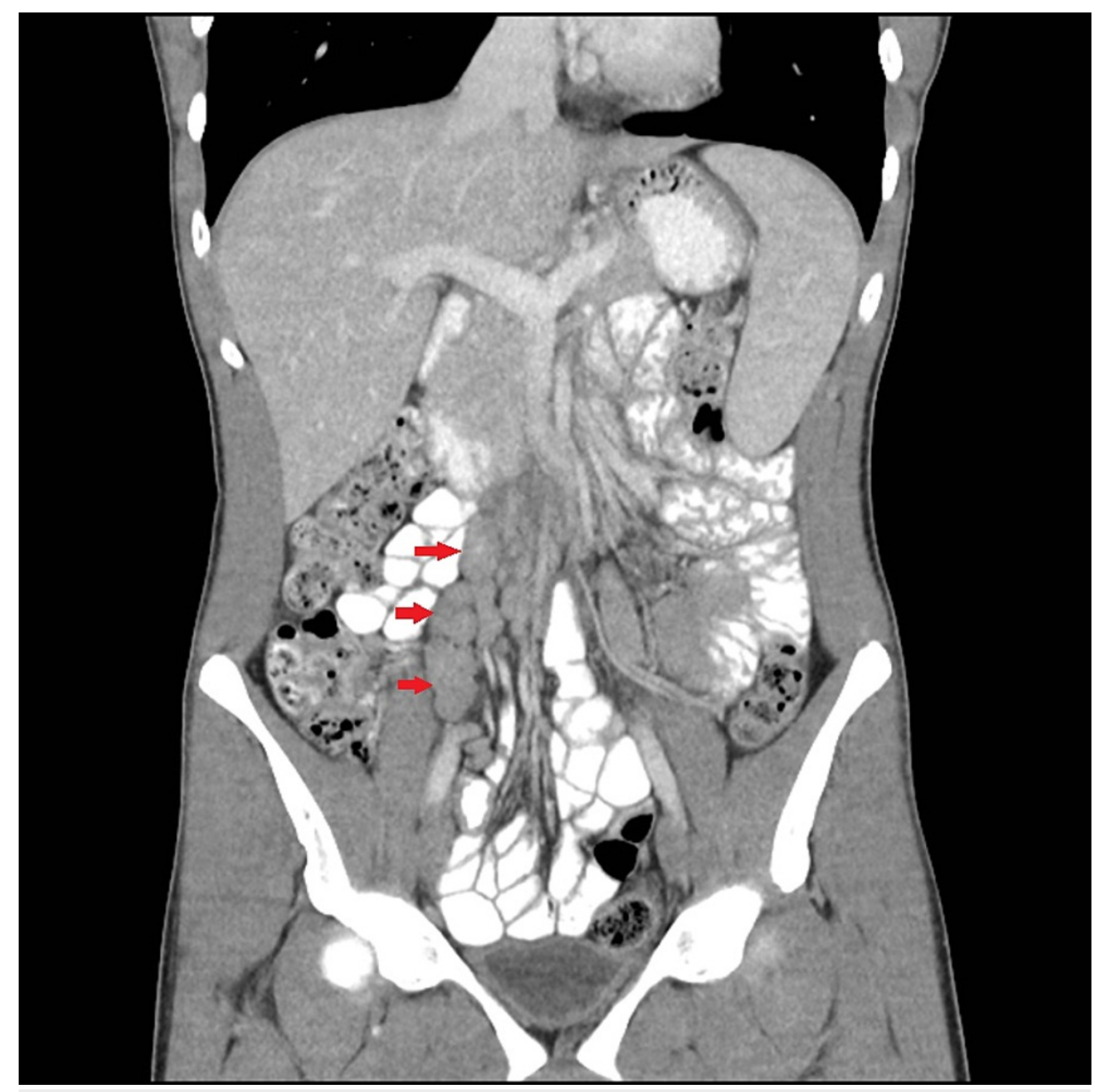

FIGURE 2: Coronal reformatted image from CT of the abdomen and pelvis with oral and IV contrast demonstrates a cluster of enlarged mesenteric lymph nodes in the right lower quadrant (red arrows).

The workup for bacterial etiology was negative. Viral panel for common respiratory pathogens including influenza A, influenza B, adenovirus, rhinovirus, respiratory syncytial virus, and human metapneumovirus returned negative. A nasopharyngeal swab for SARS-CoV-2 by Xpert ${ }^{\circledR}$ Xpress SARS-CoV-2 reverse transcriptase-polymerase chain reaction (Cepheid Inc., Sunnyvale, CA) was positive. Due to the lack of overt flu-like symptoms or respiratory distress, the patient did not receive any medical therapy for SARS-CoV-2. The patient remained in quarantine for 14 days and was subsequently released from isolation. He was reevaluated seven weeks later at which point his abdominal pain was resolved. He remained free of fever, respiratory, or gastrointestinal symptoms. Physical examination of the abdomen and testes/scrotum was normal. A repeat CT scan seven weeks later showed persistent mesenteric lymphadenopathy (Figure 3). 


\section{Cureus}

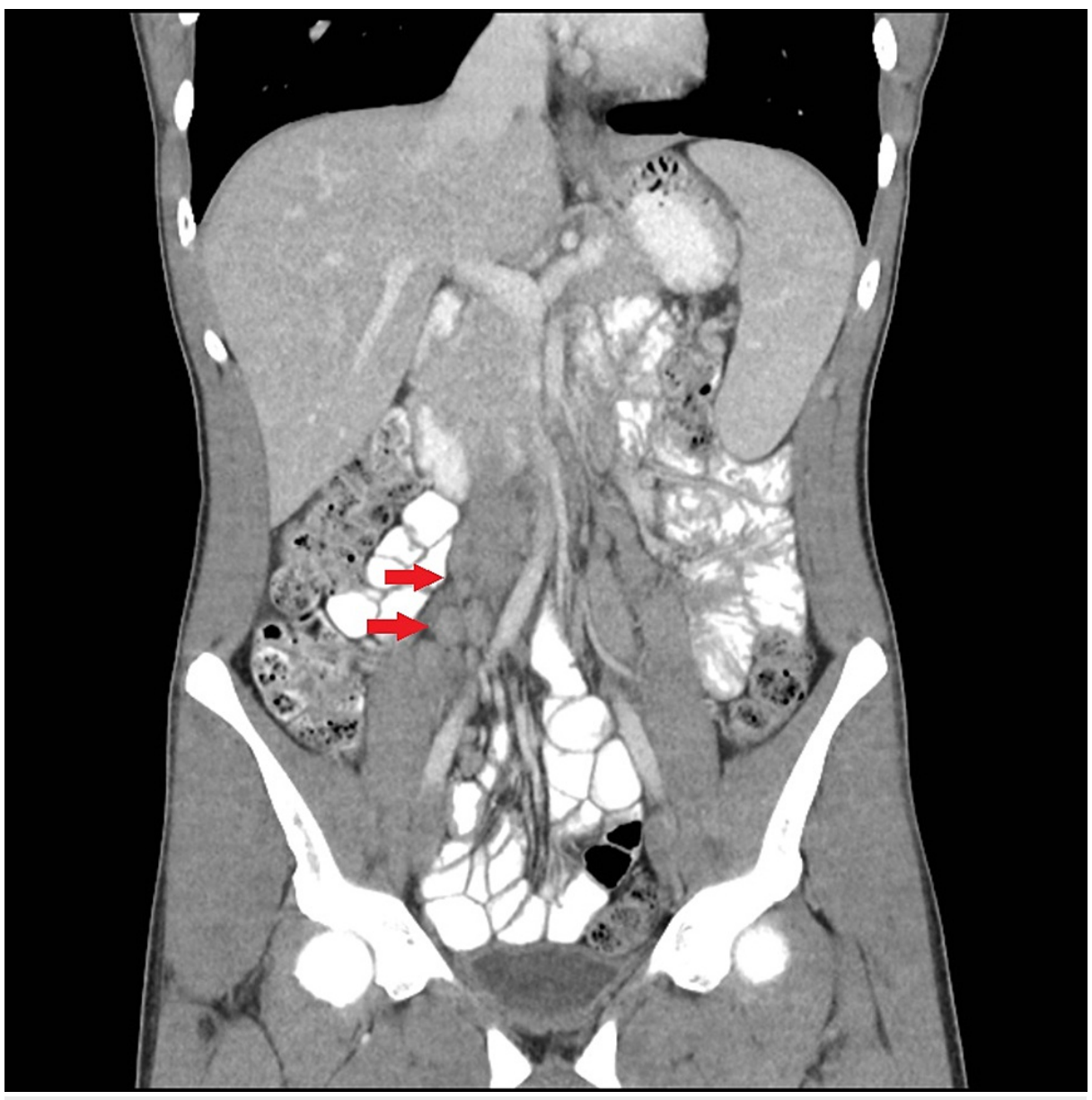

FIGURE 3: Coronal reconstructed image from CT abdomen and pelvis performed seven weeks after the initial CT shows stable enlarged mesenteric lymph nodes in the right lower quadrant (red arrows).

Repeat COVID-19 testing was not performed at this time due to limited testing availability and due to the patient's lack of symptoms. CT scan performed four months after initial diagnosis showed resolution of the lymphadenopathy.

\section{Discussion}

Acute mesenteric lymphadenitis is a self-limiting inflammatory condition typically occurring in children, adolescents, and young adults [1]. On imaging, it often presents as right-sided lymphadenopathy and may often be associated with the underlying inflammatory or infectious disease process. Reports of mesenteric adenitis have been previously reported in the pediatric population with severe COVID-19 infection who developed multisystem inflammatory syndrome in children (MIS-C), a condition characterized by systemic hyper-inflammation with multiorgan damage. A retrospective analysis of 44 pediatric patients showed that $84.1 \%$ of patients with MIS-C presented with gastrointestinal (GI) symptoms with $100 \%$ accompanied by fever and $25 \%$ of patients required supplemental oxygen [2]. Two patients were noted to have mesenteric lymphadenopathy [2]. In contrast, mesenteric adenitis has not been frequently reported among adults, especially among asymptomatic patients. Remarkably, our patient had no respiratory or gastrointestinal symptoms apart from mild abdominal and testicular pain. Further workup regarding painless lymphadenopathy was not performed as his lymphadenopathy eventually resolved in the months after his COVID-19 diagnosis. Testicular pain and painless mesenteric lymphadenopathy have been rarely reported as presenting symptoms of COVID-19 infection in the literature and may be due to persistent immune stimulation $[3,4,5]$. In such patients, the significance of prolonged lymphadenopathy raises the possibility of persistent infection with concern for asymptomatic spread. Repeat testing should be ordered in such patients for continued observation. Additionally, the long-term implications of presumed immune stimulation causing lymph node enlargement are unknown and warrant long-term observation in such patients.

\section{Conclusions}

While the frequency of atypical presentation of COVID-19 remains unknown, healthcare providers must 
continue to remain vigilant and consider COVID-19 as a differential diagnosis in any patient presenting to the emergency department despite the lack of respiratory and gastrointestinal symptoms. Considerations ought to be given to testing of such atypical or asymptomatic patients to avoid further community spread. Long-term implications of presumed immune stimulation causing lymph node enlargement are unknown and require long-term observation.

\section{Additional Information \\ Disclosures}

Human subjects: Consent was obtained or waived by all participants in this study. Conflicts of interest: In compliance with the ICMJE uniform disclosure form, all authors declare the following: Payment/services info: All authors have declared that no financial support was received from any organization for the submitted work. Financial relationships: All authors have declared that they have no financial relationships at present or within the previous three years with any organizations that might have an interest in the submitted work. Other relationships: All authors have declared that there are no other relationships or activities that could appear to have influenced the submitted work.

\section{Acknowledgements}

We would like to thank Dr. Nitish Nandu for his assistance in obtaining radiologic images for the case report.

\section{References}

1. Helbling R, Conficconi E, Wyttenbach M, et al.: Acute nonspecific mesenteric lymphadenitis: more than "no need for surgery". Biomed Res Int. 2017, 2017:9784565. 10.1155/2017/9784565

2. Miller J, Cantor A, Zachariah P, Ahn D, Martinez M, Margolis KG: Gastrointestinal symptoms as a major presentation component of a novel multisystem inflammatory syndrome in children that is related to coronavirus disease 2019: a single center experience of 44 cases. Gastroenterology. 2020, 159:1571-1574.e2. 10.1053/j.gastro.2020.05.079

3. La Marca A, Busani S, Donno V, Guaraldi G, Ligabue G, Girardis M: Testicular pain as an unusual presentation of COVID-19: a brief review of SARS-CoV-2 and the testis. Reprod Biomed Online. 2020, 41:903-6. 10.1016/j.rbmo.2020.07.017

4. Kim J, Thomsen T, Sell N, Goldsmith AJ: Abdominal and testicular pain: an atypical presentation of COVID19. Am J Emerg Med. 2020, 38:1542.e1-3. 10.1016/j.ajem.2020.03.052

5. Wani I, Tantray H, Agha N, et al.: Mesenteric lymphadenopathy in COVID-19 infection (Jammu and Kashmir sign) as a presenting sign in adult: case report. IJS: Short Reports. 2020, 5:e10. 10.1097/SR9.0000000000000010 